\title{
DENSITY THEOREMS FOR POWER SERIES AND COMPLETE SETS
}

\author{
BY \\ R. P. BOAS, JR.
}

1. Introduction. The problems considered in this paper are the completeness of sets of the form $\left\{t^{\lambda_{n}} e^{-c t}\right\}\left(c>0, \lambda_{n}>0\right)$ in $(0, \infty)$ (where the $\lambda_{n}{ }^{\prime}$ 's are not necessarily integers) and the analytic continuation of lacunary power series $\sum c_{n} z^{\lambda_{n}}$ (where the $\lambda_{n}$ 's are integers).

Let $n(t)=n_{\lambda}(t)$ denote the number of $\lambda_{n}$ not exceeding $t$. W. H. J. Fuchs [3] (1) proved that $\left\{t^{\lambda_{n}} e^{-c t}\right\}$ is complete with respect to $L^{p}, 1 \leqq p \leqq \infty$, if there is a constant $A$ such that $n(t) \geqq t / 2-A$. I shall prove by a different method th " it is sufficient to have

$$
n(t)-t / 2 \geqq-t \delta(t),
$$

where $\int^{\infty} t^{-1} \delta(t) d t$ converges. Fuchs has pointed out to me that the same result is obtainable by his original method; in addition, since the present paper was written, a paper [3a] by Fuchs has appeared, establishing the result (stronger if the $\lambda_{n}$ satisfy $\left.\lambda_{n+1}-\lambda_{n} \geqq \epsilon>0\right)$ that if $\lambda_{n+1}-\lambda_{n} \geqq \epsilon>0,\left\{t^{\lambda_{n}} e^{-c t}\right\}$ is complete with respect to $L^{2}$ if and only if

$$
\int_{1}^{\infty} r^{-2} \psi(r) d r=\infty, \quad \psi(r)=\exp \left\{2 \sum_{\lambda_{n}<r} \lambda_{n}^{-1}\right\},
$$

and that (1.2) is sufficient for completeness with respect to $L^{p}, p \neq 2, p \geqq 1$. However, the proof given in the present paper is somewhat simpler than either of Fuchs's proofs.

Fuchs also showed [3] that if the set $\{n\}$ of all positive integers is divided into two complementary subsequences $\left\{\lambda_{n}\right\}$ and $\left\{\mu_{n}\right\}$, then at least one of $\left\{t^{\lambda_{n}} e^{-c t}\right\}$ and $\left\{t^{\mu_{n}} e^{-c t}\right\}$ is complete. (This is a trivial consequence of his later result [3a].) I shall show that $\{n\}$ can be replaced by any sequence $\left\{a_{n}\right\}$ such that $n_{a}(t)>t-t \delta(t)$ with $\int^{\infty} t^{-1} \delta(t) d t$ convergent (see Theorem 4 , where an extension to $k$ subsequences is given). This is a corollary of Fuchs's results in [3a] if $a_{n+1}-a_{n} \geqq \epsilon>0$.

The completeness of sets $\left\{t^{\lambda_{n}} e^{-c t}\right\}$ is equivalent to the completeness of various other sets. For example, the Fourier cosine transform leads (as was pointed out to me by $\mathrm{H}$. Pollard) to the set $\left\{(\cos x)^{\lambda_{n}} \cos \lambda_{n} x\right\}$ in $(0, \pi / 2)$; the Mellin transform leads to the set $\left\{\Gamma\left(\lambda_{n}+i t\right)\right\}$ on $(-\infty, \infty)$.

Presented to the Society, September 15, 1945, under the title Some complete sets of functions; received by the editors October 23, 1945.

(1) Numbers in brackets refer to the references at the end of the paper. 
Almost the same reasoning as is used for the completeness problem leads to new criteria for the uniqueness of the solution of the generalized moment problem

$$
\mu_{n}=\int_{0}^{\infty} t^{\lambda_{n}} d \alpha(t)
$$

$\alpha(t)$ nondecreasing (see $\S 5$ ).

Let $f(z)$ have a power series of the form

$$
\sum_{n=0}^{\infty} c_{n} z^{\lambda_{n}}
$$

where the $\lambda_{n}$ are integers and

$$
\limsup _{n \rightarrow \infty} n_{\lambda}(r) / r=D .
$$

If $f(z)$ is defined by (1.3) for small $|z|$ and by analytic continuation (if possible) for large $|z|$, Mandelbrojt has shown that, in every angle with vertex at the origin and opening $2 \alpha>2 \pi D, f(z)$ (if not a constant) either has a singular point or is unbounded. Mandelbrojt's result applies to Dirichlet series, and is more general in other ways; but it requires the strict inequality $\left({ }^{2}\right)$ $\alpha>\pi D$. I shall give some results in which, if (1.4) is somewhat strengthened, $\alpha=\pi D$ is permissible. In the first place, (1.4) can be replaced by

$$
n(r) \leqq r\left(\pi^{-1} \alpha-\epsilon(r)\right),
$$

where $\int^{\infty} r^{-1} \epsilon(r) d r$ diverges; (1.4) with $\alpha>\pi D$ is the case where $\epsilon(r) \geqq \epsilon>0$. In the second place, when $\alpha=\pi / 2$, results on complete sets can be applied to show that $f(z)$ (if not constant) either has a singular point or is unbounded in every half-plane containing $z=0$ in its interior, provided only that

$$
n(r) \leqq r(1 / 2+\delta(r)),
$$

where $\delta(r)$ has the same properties as in (1.1). By applying Fuchs's later result [3a] instead of the results of the present paper, (1.6) can be replaced by (1.2); either (1.6) or (1.2) requires less of $n(r)$ than (1.4), but implies a little less about $f(z)$. These results for $\alpha=\pi / 2$ imply a corresponding improvement of results of Mandelbrojt and Ulrich [6] on a generalization of quasi-analyticity.

The power series result for $\alpha=\pi / 2$ is not only a consequence of the completeness of $\left\{t^{\lambda_{n}} e^{-c t}\right\}$, but also implies it. It is interesting to observe that, as Fuchs showed [3], the completeness of $\left\{t^{\lambda_{n}} e^{-c t}\right\}$ is also equivalent to the following statement about differences: if $\left\{a_{n}\right\}$ is a sequence such that $a_{n}=o(n)$

(2) Mandelbrojt [4] states the theorem with $\alpha \geqq \pi D$, but this is evidently an oversight, as his proof shows. 
and $\Delta^{\lambda_{n}} a_{0}=0$, then $\left\{a_{n}\right\}$ is constant $\left({ }^{3}\right)$.

There are also decomposition theorems for power series analogous to those for complete sets. For example, let $\{n\}=\left\{\lambda_{n}\right\}+\left\{\mu_{n}\right\}, \alpha_{1}>0, \alpha_{2}>0, \alpha_{1}+\alpha_{2}$ $>\pi$. If $f_{1}(z)=\sum c_{n} z^{\lambda_{n}}$ and $f_{2}(z)=\sum \gamma_{n} z^{\mu_{n}}$ and if we take any two angles of openings $2 \alpha_{1}, 2 \alpha_{2}$, with vertices at the origin, then one of $f_{1}(z), f_{2}(z)$ has a singular point, is unbounded, or is a constant in the corresponding angle. A stronger result can be obtained if we start from a sequence which already possesses gaps.

The theorems of this paper depend on some uniqueness results for functions analytic in a half-plane; these will be given first.

2. Lemmas on entire functions. We begin with some properties of special entire functions.

LEMMA 2.1. Let $\left\{\lambda_{n}\right\}$ be an increasing sequence of positive numbers and let $n(r)$ denote the number of $\lambda_{n}$ not exceeding $r$. Let $\delta(t)$ be a positive function vanishing near $t=0$. Let $c>0$ and

$$
n(t) \geqq c t-t \delta(t), \quad n(t)=O(t), \quad t \rightarrow \infty .
$$

Then the product

$$
\phi(z)=\prod_{n=1}^{\infty}\left(1-. z^{2} / \lambda_{n}^{2}\right)
$$

converges and there are constants $A_{1}, A_{2}$ such that for $r>0$

$$
\log \left|\phi\left(r e^{i \theta}\right)\right| \geqq c \pi r|\sin \theta|-A_{1} r \delta_{1}(r), \quad \pi / 4 \leqq|\theta| \leqq \pi / 2,
$$

where $r \delta_{1}(r)$ is nondecreasing and $\int^{\infty} r^{-1} \delta_{1}(r) d r$ converges or diverges with $\int^{\infty} r^{-1} \delta(r) d r$, while for an unbounded sequence of $r$ 's,

$$
\log \left|\phi\left(r e^{i \theta}\right)\right| \geqq-A_{2} r .
$$

The convergence of the product follows from (2.2), which also shows, since $\phi(z)$ is even, that it is a function of order 1 and finite type; (2.5) is a well known result for such functions $[10$, p. 276].

We have, if $z$ is not real,

$$
\begin{aligned}
\log \phi(z) & =\sum_{n=1}^{\infty} \log \left(1-z^{2} / \lambda_{n}^{2}\right)=\int_{0}^{\infty} \log \left(1-z^{2} / t^{2}\right) d n(t) \\
& =\lim _{R \rightarrow \infty}\left\{n(R) \log \left(1-z^{2} / R^{2}\right)-2 z^{2} \int_{0}^{R} \frac{n(t) d t}{t\left(t^{2}-z^{2}\right)}\right\} \\
& =-2 z^{2} \int_{0}^{\infty} \frac{n(t) d t}{t\left(t^{2}-z^{2}\right)} ;
\end{aligned}
$$

(3) The case $\left\{\lambda_{n}\right\}=2 n$ was found independently by Agnew [1]; a simpler proof was given by Pollard [8]; the equivalence of "power series," "completeness," and "difference" theorems has been given a simplified proof by Boas and Pollard [2a]. 
the "integrated term" disappears by (2.2), since $\log \left(1-z^{2} / R^{2}\right)=O\left(R^{-2}\right)$ as $R \rightarrow \infty$. Hence

$$
\log |\phi(z)|=-2 \Re\left\{\int_{0}^{\infty} \frac{n(t)}{t} \frac{z^{2}}{t^{2}-z^{2}} d t\right\} .
$$

Let $n(t)=c t+t \zeta(t)$, where $\zeta(t) \geqq-\delta(t)$. Then

$$
\begin{aligned}
\log |\phi(z)| & =-2 c \Re\left\{\int_{0}^{\infty} \frac{z^{2}}{t^{2}-z^{2}} d t\right\}-2 \int_{0}^{\infty} \zeta(t) \Re\left\{\frac{z^{2}}{t^{2}-z^{2}}\right\} d t \\
& =I_{1}+I_{2},
\end{aligned}
$$

say. We have

$$
I_{1}=\pi c|y| \text {. }
$$

Also,

$$
\Re\left\{\frac{z^{2}}{t^{2}-z^{2}}\right\}=-r^{2} \frac{r^{2}-t^{2} \cos 2 \theta}{t^{4}-2 t^{2} r^{2} \cos 2 \theta+r^{4}}
$$

and so

$$
I_{2}=2 r^{2} \int_{0}^{\infty} \zeta(t) \frac{r^{2}-t^{2} \cos 2 \dot{\theta}}{t^{4}-2 t^{2} r^{2} \cos 2 \theta+r^{4}} d t .
$$

If $\pi / 4 \leqq|\theta| \leqq \pi / 2, \cos 2 \theta \leqq 0$ and so we have.

where

$$
I_{2} \geqq-2 r^{2} \int_{0}^{\infty} \delta(t) \frac{t^{2}+r^{2}}{t^{4}+r^{4}} \dot{d t}=-r \delta_{1}(r),
$$

$$
\delta_{1}(r)=2 r \int_{0}^{\infty} \delta(t) \frac{t^{2}+r^{2}}{t^{4}+r^{4}} d t
$$

so that $r \delta_{1}(r)$ is nondecreasing. We have

$$
\begin{aligned}
\int_{0}^{\infty} r^{-1} \delta_{1}(r) d r & =2 \int_{0}^{\infty} d r \int_{0}^{\infty} \delta(t) \frac{t^{2}+r^{2}}{t^{4}+r^{4}} d t \\
& =2 \int_{0}^{\infty} \delta(t) d t \int_{0}^{\infty} \frac{t^{2}+r^{2}}{t^{4}+r^{4}} d t \\
& =C \int_{0}^{\infty} t^{-1} \delta(t) d t,
\end{aligned}
$$

where $C$ is a positive constant; consequently $\int^{\infty} r^{-1} \delta_{1}(r) d r$ and $\int^{\infty} t^{-1} \delta(t) d t$ converge or diverge together. 
LEMMA 2.7. With the hypotheses of Lemma 2.1, except that (2.2) is replaced by

$$
n(t) \geqq c t+t \delta(t),
$$

we have for $r>0$

$$
\log \left|\phi\left(r e^{i \theta}\right)\right| \geqq c \pi r|\sin \theta|+A_{3} r \delta_{2}(r),
$$$$
\pi / 4 \leqq|\theta| \leqq \pi / 2,
$$

where $\delta_{2}(r)$ has the same properties as $\delta_{1}(r)$, and we have (2.5) for an unbounded sequence of $r$ 's.

The proof is the same as for Lemma 2.1, through (2.6). We then have

$$
\begin{aligned}
I_{2} & \geqq 2 r^{2} \int_{0}^{\infty} \delta(t) \frac{r^{2}-t^{2} \cos 2 \theta}{t^{4}-2 t^{2} \cos 2 \theta+r^{4}} d t \\
& \geqq 2 r^{4} \int_{0}^{\infty} \frac{\delta(t)}{\left(t^{2}+r^{2}\right)^{2}} d t=r \delta_{2}(r), \\
\delta_{2}(r) & =2 r^{3} \int_{0}^{\infty} \frac{\delta(t) d t}{\left(t^{2}+r^{2}\right)^{2}} .
\end{aligned}
$$

Just as before, it follows that $\int{ }^{\infty} r^{-1} \delta_{2}(r) d r$ and $\int^{\infty} r^{-1} \delta(r) d r$ converge or diverge together.

LEMma 2.10. If $\eta(r)$ is nonincreasing, $r \eta(r) \uparrow \infty$ as $r \uparrow \infty$, and $\int^{\infty} t^{-1} \eta(t) d t$ converges, there is an entire function $\psi(z)$ such that, for $|\theta| \leqq \pi / 2$,

$$
\log \left|\psi\left(r e^{i \theta}\right)\right| \geqq r \eta(r) / 3, \quad r \geqq r_{0},
$$

where $r_{0}$ is a positive constant.

Let $\left\{\lambda_{n}\right\}$ be an increasing sequence of positive numbers such that

$$
A r \eta(r) \geqq n_{\lambda}(r) \geqq r \eta(r), \quad r \geqq b>0,
$$

where $A$ is a constant greater than 1 . We shall show that

$$
\psi(z)=\prod_{n=1}^{\infty}\left(1+z / \lambda_{n}\right)
$$

has the required properties.

We have, as $R \rightarrow \infty$,

$$
\begin{aligned}
\sum_{\lambda_{n} \leqq R} 1 / \lambda_{n} & =\int_{0}^{R} t^{-1} d n(t)=R^{-1} n(R)+\int_{0}^{R} t^{-2} n(t) d t \\
& \leqq A \eta(R)+\int_{\lambda_{1}}^{R} t^{-1} \eta(t) d t=O(1) ;
\end{aligned}
$$

hence $\sum 1 / \lambda_{n}$ converges, and so $\psi(z)$ is entire. 
We then have

$$
\log \psi(z)=\sum_{n=1}^{\infty} \log \left(1+z / \lambda_{n}\right)=\int_{0}^{\infty} \log (1+z / t) d n(t)=\int_{0}^{\infty} \frac{z n(t)}{t(t+z)} d t,
$$

and consequently, if $|\theta| \leqq \pi / 2$,

$$
\begin{aligned}
\log |\psi(z)| & =\Re\{\log \psi(z)\}=\int_{0}^{\infty} \frac{n(t)}{t} \frac{r^{2}+r t \cos \theta}{r^{2}+2 r t \cos \theta+t^{2}} d t & \\
& \geqq r^{2} \int_{0}^{\infty} \frac{n(t) d t}{t(r+t)^{2}} \geqq r^{2} \int_{b}^{r} \frac{\eta(t) d t}{(r+t)^{2}} & \\
& \geqq(r-b) \eta(r) / 2>r \eta(r) / 3, & r \geqq 2 b .
\end{aligned}
$$

3. Analytic functions in a half-plane. We can now prove the uniqueness theorems which we need. let

Theorem 1. Let $F(z)$ be analytic in $x>0$ and continuous in $x \geqq 0$. For $x \geqq 0$,

$$
\log |F(z)| \leqq m x \log x+A x+\sigma(r), \quad m>0,
$$

where $A$ is a constant, $\sigma(r)$ is nondecreasing and $\int^{\infty} t^{-2} \sigma(t) d t$ converges. Let $F\left(\lambda_{n}\right)=0$, where $\lambda_{n}>0$ and

$$
n_{\lambda}(t) \geqq m t / 2-t \delta(t),
$$

$\delta(t) \downarrow 0$ and $\int^{\infty} t^{-1} \delta(t) d t$ converges. Then $F(z) \equiv 0$.

Let $\phi(z)$ be the function of Lemma 2.1, with the given sequence $\left\{\lambda_{n}\right\}$ and $c=m / 2$. (If $n(t) \neq O(t)$, we can discard $\lambda_{n}$ 's until $n(t)=O(t)$ without affecting (3.2).) Let $\eta(t)$ be nonincreasing, $t \eta(t) \uparrow \infty$, with $\int^{\infty} t^{-1} \eta(t) d t$ convergent and $\eta(t)>3 A_{1} \delta_{1}(t)+3 t^{-1} \sigma(t)$, where $A_{1}$ and $\delta_{1}$ are the quantities appearing in (2.4). Let $\psi(z)$ be the function of Lemma 2.10 , and consider the function

$$
H(z)=\frac{F(z)}{B\{\Gamma(z)\}^{m} \phi(z) \psi(z)},
$$

where $B$ is a constant, to be chosen in a moment. Since the zeros of $\phi(z)$ in $x>0$ are cancelled by the zeros of $F(z), H(z)$ is analytic in $x>0$ and continuous in $x \geqq 0$. We then have, for $r>r_{1}$, where $r_{1}>r_{0}$ is a suitable constant, $\log |H(z)| \leqq m r \cos \theta \log r+m r \cos \theta \log \cos \theta+A r \cos \theta+\sigma(r)$

$-m r \cos \theta \log r+m r \theta \sin \theta-\log \left|\phi\left(r e^{i \theta}\right)\right|-r \eta(r) / 2-\log B$

$\leqq m r \cos \theta(A+\log \cos \theta)+m r \theta \sin \theta+\sigma(r)$

$$
-r \eta(r) / 2-\log \left|\phi\left(r e^{i \theta}\right)\right|-\log B \text {. }
$$


Hence, for $\pi / 4 \leqq|\theta| \leqq \pi / 2$ and $r>r_{1}$,

$\log |H(z)| \leqq m r \cos \theta(A+\log \cos \theta)+m r|\sin \theta|(|\theta|-\pi / 2)$

$$
\begin{aligned}
& +\sigma(r)+A_{1} r \delta_{1}(r)-r \eta(r) / 2-\log B \\
\leqq & m r \cos \theta(A+\log \cos \theta)-\log B .
\end{aligned}
$$

If $B$ is chosen larger than $\max |B H(z)|$ for $|z| \leqq r_{1}, x \geqq 0$, we have $\log |H(z)|$ $<0$ for $|z|<r_{1}$, and so

$$
\log |H(z)| \leqq m r \cos \theta(A+\log \cos \theta), \quad r \geqq 0, \pi / 4 \leqq|\theta| \leqq \pi / 2 .
$$

For $0 \leqq|\theta| \leqq \pi / 2$ and for an unbounded sequence of values of $r$ we also have

$$
\log |H(z)| \leqq C r,
$$

where $C$ is a constant.

Now let $K_{\omega}(z)=H(z) e^{m \omega z}, \omega>0$. Then by (3.3), for $\pi / 4 \leqq|\theta| \leqq \pi / 2$,

$$
\log \left|K_{\omega}(z)\right| \leqq m r \cos \theta(\log \cos \theta+A+\omega)
$$

while

$$
\log \left|K_{\omega}(z)\right| \leqq(C+\omega) r, \quad 0 \leqq|\theta| \leqq \pi / 2,
$$

for an unbounded sequence of values of $r$.

If $\theta_{0}$ is chosen so that $\cos \theta_{0}<e^{-\omega}$, we then have $\log \left|K_{\omega}(z)\right| \leqq 0$ for $\theta= \pm \theta_{0}$, $\theta_{0}<\pi / 2$, and so, by a well known Phragmén-Lindelöf theorem $[10$, p. 177], $\log \left|K_{\omega}(z)\right| \leqq 0$ for $0 \leqq|\theta| \leqq \theta_{0}$. In particular, then,

$$
\left|H(x) e^{\omega x}\right| \leqq 1
$$

for $x>0$. Letting $\omega \rightarrow \infty$, we obtain $H(z) \equiv 0$. Hence $F(z) \equiv 0$.

THEOREM 2. Let $F(z)$ be analytic in $x>0$. For $x \geqq 1$, let

$$
\log |F(x+i y)| \leqq k|y|+\sigma(r),
$$

where $\int^{\infty} t^{-2} \sigma(t) d t$ converges. Let $F\left(\mu_{n}\right)=0$, where $\mu_{n}>0$ and

$$
n_{\mu}(t) \geqq(k / \pi) t+t \delta(t),
$$

$\delta(t)$ is nonincreasing, and $\int^{\infty} t^{-1} \delta(t) d t$ diverges. Then $F(z) \equiv 0$.

Let $\phi(z)$ be the function of Lemma 2.7, with $\mu_{n}$ replacing $\lambda_{n}$. In the part of $x \geqq 1$ where $\pi / 4 \leqq|\theta| \leqq \pi / 2$, we have

$$
\log |F(z) / \phi(z)| \leqq \sigma(r)-A_{3} r \delta_{2}(r),
$$

and

$$
\log |F(z) / \phi(z)|=O(r)
$$

on a sequence of semicircles of unbounded radii. Applying Carleman's theorem $[10$, p. 130$]$ to $F(z) / \phi(z)$ in this half-plane, we obtain 


$$
\begin{aligned}
0 & \leqq O(1)+\pi^{-1} \int_{1}^{R}\left\{y^{-2} \sigma(y)-A_{3} y^{-1} \delta_{2}(y)\right\} d y \\
& =O(1)-A_{3} \int_{1}^{R} y^{-1} \delta_{2}(y) d y .
\end{aligned}
$$

Since $\int^{\infty} y^{-1} \delta_{2}(y) d y$ diverges, this leads to a contradiction unless $F(z) \equiv 0$.

4. Completeness of sets $\left\{t^{\lambda_{n}} e^{-c t}\right\}$. We can always suppose that $c=1$, by making a change of variable if necessary. We say that a set of functions $\left\{f_{n}(t)\right\}_{n=1}^{\infty}$ is complete with respect to a class $C$ if

$$
\int_{0}^{\infty} f_{n}(t) g(t) d t=0, \quad n=1,2, \cdots ; g \in C,
$$

implies $g(t)=0$ in $C$.

THEOREM 3(4). If $\left\{\lambda_{n}\right\}$ is an increasing positive sequence with

$$
n(t) \geqq t / 2-t \delta(t), \quad \int^{\infty} t^{-1} \delta(t) d t<\infty,
$$

$\delta(t)$ nonincreasing, then the set $\left\{t^{\lambda_{n}} e^{-t}\right\}$ is complete with respect to every $L^{p}(0, \infty)$, $1 \leqq p \leqq \infty$.

We have to show that if

$$
\int_{0}^{\infty} e^{-t} t^{\lambda_{n}} f(t) d t=0, \quad n=1,2, \cdots ; f(t) \in L^{p}(0, \infty),
$$

then $f(t)=0$ almost everywhere. We consider the function

$$
F(z)=\int_{0}^{\infty} e^{-t} t^{z} f(t) d t
$$

and show that, if $F\left(\lambda_{n}\right)=0(n=1,2, \cdots)$, then $F(z) \equiv 0$. That $f(\dot{t})=0$ almost everywhere then follows from the uniqueness theorem for Mellin transforms.

$F(z)$ is analytic in $x>0$ and continuous in $x \geqq 0$. If $p=\infty$, we have $|f(t)| \leqq M$ and so

$$
|F(z)| \leqq \int_{0}^{\infty} e^{-t} t^{x} d t=\Gamma(x+1)
$$

If $p=1$, let $\int_{0}^{\infty}|f(t)| d t=M$; then

$$
|F(z)| \leqq M \sup _{0 \leqq t<\infty} e^{-t} t^{x}=e^{-x} x^{x}
$$

If $1<p<\infty$, let $p^{\prime}=p /(p-1)$; then, by Hölder's inequality,

(4) A result of Fuchs [3a], stronger when $\lambda_{n+1}-\lambda_{n} \geqq \epsilon>0$, is quoted in $\$ 1$. 


$$
\begin{aligned}
|F(z)| & \leqq\left\{\int_{0}^{\infty}|f(t)| p d t\right\}^{1 / p}\left\{\int_{0}^{\infty} e^{\left.-p^{\prime} t t^{p^{\prime} x} d t\right\}^{1 / p^{\prime}}}\right. \\
& =M\left\{\Gamma\left(p^{\prime} x+1\right)\right\}^{1 / p^{\prime}} .
\end{aligned}
$$

Using Stirling's formula when $p>1$, we thus have in all cases

$$
\log |F(z)| \leqq x \log x-x+B \log x
$$

where $B$ depends only on $p$.

$F(z)$ thus satisfies the hypotheses of Theorem 1 , with $m=1, \sigma(r)=B \log r$. Consequently $F(z) \equiv 0$.

THEOREM 4. Let $\left\{a_{n}\right\}$ be a real positive sequence with

$$
n_{a}(t)>m t / 2-t \delta(t)
$$

where $m$ is an integer greater than 1 and $\delta(t)$ satisfies the conditions of Theorem 3 . Then if $\left\{a_{n}\right\}$ is divided into $m$ exhaustive and nonoverlapping sequences $\left\{\lambda_{n}\right\}, \cdots,\left\{{ }_{m} \lambda_{n}\right\}$, at least one of the sets $\left\{t^{k \lambda_{n}} e^{-t}\right\}$ is complete with respect to $L^{p}(0, \infty)(1 \leqq p \leqq \infty)$.

Suppose that the first $m-1$ of the sets are not complete in a specified $L^{p}$; then there exist $m-1$ functions $\phi_{k}(t)$ of $L^{p}$ such that

$$
\begin{aligned}
F_{k}(z) & =\int_{0}^{\infty} t^{z} e^{-t} \phi_{k}(t) d t \not \equiv 0, \\
F_{k}\left({ }_{k} \lambda_{n}\right) & =0, \quad n=1,2, \cdots ; k=1,2, \cdots, m-1 .
\end{aligned}
$$

If $\phi_{m}(t)$ is orthogonal to all the functions $t^{m \lambda_{n}} e^{-t}$, let

$$
F_{m}(z)=\int_{0}^{\infty} t^{z} e^{-t} \phi_{m}(t) d t
$$

and let

$$
F(z)=F_{1}(z) F_{2}(z) \cdots F_{m}(z) .
$$

Then $F\left(a_{n}\right)=0(n=1,2, \cdots)$. By the reasoning of Theorem 3 , we have

$$
\log |F(z)| \leqq m(x \log x-x+B \log x), \quad x \geqq 0 .
$$

$F(z)$ now satisfies the hypotheses of Theorem 1 , and consequently $F(z) \equiv 0$. Hence $F_{m}(z) \equiv 0$, and so the set $\left\{t^{m^{\lambda_{n}} e^{-t}}\right\}$ is complete.

5. A generalized moment problem. Let $\left\{\mu_{n}\right\}$ be a sequence of positive numbers such that

$$
\mu_{n}=\int_{0}^{\infty} t^{\lambda_{n}} d \alpha(t)
$$


for at least one nondecreasing $\alpha(t)$. The problem is to find conditions on $\left\{\mu_{n}\right\}$ which imply that $\alpha(t)$ is unique if normalized $(\alpha(0+)=0, \alpha(t)$ $=\{\alpha(t+)+\alpha(t-)\} / 2)$. The following theorem gives a sufficient condition for uniqueness which is better than conditions given previously [2] for nonintegral $\lambda_{n}$, though weakęr than Carleman's criterion $\left[9\right.$, p. 20] for $\lambda_{n}=n$.

ThEOREM 5. Let $\left\{\lambda_{n}\right\}$ be an increasing sequence such that

$$
\lambda_{n} \leqq \lambda_{n-1}\left(1+1 / \log \lambda_{n-1}\right)
$$

and $n_{\lambda}(t) \geqq t(1-\delta(t))$, where $\delta(t)$ satisfies the conditions of Theorem 3 . Then two normalized nondecreasing functions $\alpha_{1}(t), \alpha_{2}(t)$ satisfying

$$
\mu_{n}=\int_{0}^{\infty} t^{\lambda_{n}} d \alpha_{j}(t), \quad j=1,2,
$$

are identical if there is a constant $\sigma$ such that

$$
\mu_{n}^{1 /\left(2 \lambda_{n}\right)} \leqq \sigma \lambda_{n}, \quad n=1,2, \cdots .
$$

Suppose that $\alpha_{1}(t)$ and $\alpha_{2}(t)$ satisfy (5.3), and let

$$
F(z)=\int_{0}^{\infty} t^{z} d\left\{\alpha_{1}(t)-\alpha_{2}(t)\right\},
$$

so that $F\left(\lambda_{n}\right)=0$. Then

$$
|F(z)| \leqq \int_{0}^{\infty} t^{x} d\left\{\alpha_{1}(t)+\alpha_{2}(t)\right\} ;
$$

and so $\left|F\left(\lambda_{n}+i y\right)\right| \leqq 2 \mu_{n}$ and

$$
\begin{aligned}
\log \left|F\left(\lambda_{n}+i y\right)\right| & \leqq \log 2+\log \mu_{n} \\
& \leqq \log 2+2 \lambda_{n} \log \left(\sigma \lambda_{n}\right) \\
& \leqq 2 \lambda_{n-1} \log \lambda_{n-1}+A \lambda_{n-1},
\end{aligned}
$$

where $A$ is independent of $n$. For, we may assume without loss of generality that $\lambda_{n-1} \geqq e$, and then, since

$$
\lambda_{n} \leqq \lambda_{n-1}+\lambda_{n-1} / \log \lambda_{n-1},
$$

we have

$$
\begin{aligned}
\lambda_{n} \log \left(\sigma \lambda_{n}\right) & \leqq\left(\lambda_{n-1}+\lambda_{n-1} / \log \lambda_{n-1}\right)\left(\log \lambda_{n}+\log \sigma\right) \\
& \leqq\left(\lambda_{n-1}+\lambda_{n-1} / \log \lambda_{n-1}\right)\left(\log \lambda_{n-1}+\log \left(1+1 / \log \lambda_{n-1}\right)+\log \sigma\right) \\
& \leqq\left(\lambda_{n-1}+\lambda_{n-1} / \log \lambda_{n-1}\right)\left(\log \lambda_{n-1}+\log \sigma+1 / \log \lambda_{n-1}\right) \\
& \leqq \lambda_{n-1} \log \lambda_{n-1}+\lambda_{n-1}(3+2 \log \sigma),
\end{aligned}
$$


and (5.5) follows. Hence, if $\lambda_{n-1} \leqq x<\lambda_{n}$,

$$
\begin{aligned}
\log |F(x+i y)| & \leqq \log \left|F\left(\lambda_{n}+i y\right)\right| \\
& \leqq 2 \lambda_{n-1} \log \lambda_{n-1}+A \lambda_{n-1} \\
& \leqq 2 x \log x+A x .
\end{aligned}
$$

Theorem 1 now shows that $F(z) \equiv 0$, and consequently $\alpha_{1}(t) \equiv \alpha_{2}(t)$.

6. Gap theorems for power series in half-planes. We next apply the results of $\S 4$ to power series.

THEOREM 6(5). Let

$$
f(z)=\sum_{n=0}^{\infty} a_{n} z^{\lambda_{n}}, \quad|z|<1,
$$

where $\left\{\lambda_{n}\right\}$ is an increasing sequence of positive integers such that

$$
n_{\lambda}(r) \leqq r / 2+r \delta(r) ;
$$

here $\delta(t)$ satisfies the conditions of Theorem 3 ; namely, $\delta(t)$ nonincreasing and $\int^{\infty} t^{-1} \delta(t) d t$ convergent. Then if $f(z)$ is not a constant it cannot be analytic and bounded in any half-plane having the origin as an interior point.

Theorem 6 follows, by Theorem 3 , from the following result, which establishes the equivalence of gap theorems with conclusions like that of Theorem 6 and completeness theorems for sets $\left\{t^{\lambda} e^{-c t}\right\}$.

THEOREM 7. Let $\left\{\lambda_{n}\right\}$ be a sequence of positive integers, $\left\{\mu_{n}\right\}$ the complementary sequence. The following two statements are equivalent.

(A) The set $\left\{t^{\mu_{n}} e^{-c t}\right\}$ is complete in $L^{2}(0, \infty)$.

(B) Every function $f(z)=\sum_{n=0}^{\infty} c_{n} z^{\lambda_{n}}$, not a constant, either has a singular point or is unbounded in every half-plane containing the origin in its interior.

For the application to Theorem 6, we need only note that (6.1) implies

$$
n_{\mu}(r) \geqq r / 2-r \delta_{1}(r),
$$

where $\delta_{1}(r)$ satisfies the same conditions as $\delta(r)$.

Theorem 7 is proved by Boas and Pollard [2a].

THEOREM 8. Let $\left\{c_{n}\right\}$ be a sequence of complex numbers and let $\left\{\lambda_{n}\right\},\left\{\mu_{n}\right\}$ be two complementary sequences of the positive integers. Let

$$
F_{1}(z)=\sum_{n=1}^{\infty} c_{\lambda_{n}} z^{\lambda_{n}}, \quad F_{2}(z)=\sum_{n=1}^{\infty} c_{\mu_{n}} z^{\mu_{n}} .
$$

Then, in every half-plane having the origin as an interior point, one at least of in $\$ 1$.

(b) A stronger result follows by the same reasoning from the result of Fuchs [3a] quoted 
$F_{1}(z)$ and $F_{2}(z)$ has a singular point, is unbounded, or is identically a constant.

This follows from Theorem 4, with $m=2$, together with Theorem 7 .

7. Gap theorems for power series in angles. We now establish the remainder of the theorems outlined in $\$ 1$.

Theorem 9. Let

$$
f(z)=\sum_{n=0}^{\infty} c_{n} z^{n}, \quad|z| \leqq 1,
$$

where $c_{n}=0$ except for $n=\lambda_{n}$. Let

$$
n_{\lambda}(t) \leqq \pi^{-1} \alpha t-t \delta(t), \quad \alpha<\pi,
$$

where $\delta(t)$ satisfies the conditions of Theorem 2 , namely, $\delta(t)$ nonincreasing, and $\int^{\infty} t^{-1} \delta(t) d t$ divergent. If $f(z)$ is not a constant, it is unbounded or has a singular point in every closed angle of opening $2 \alpha$, with vertex at 0.

The special case in which the upper density of $\left\{\lambda_{n}\right\}$ is less than $\alpha / \pi$ is not quite included in the result of Mandelbrojt quoted in $\$ 1$. The case $\alpha=\pi / 2$ is included in Theorem 6 .

We may suppose that the angle is $|\theta| \leqq \alpha$. Let $f(z)$ be analytic and bounded in this angle; we shall show that $f(z)$ is a constant.

We have

$$
2 \pi c_{n}=-i \int_{C} z^{-n-1} f(z) d z,
$$

where $C$ consists of the $\operatorname{arc} \alpha \leqq \theta \leqq 2 \pi-\alpha$ of the circle $|z|=1$, the line segments $\theta= \pm \alpha, 0 \leqq r \leqq R(R>1)$, and the arc $-\alpha \leqq \theta \leqq \alpha$ of $|z|=R$. The integral along the latter arc is $R^{-n} \int_{-\alpha}^{\alpha} e^{i n \theta} f\left(R e^{i \theta}\right) d \theta$, which approaches zero as $R \rightarrow \infty$ provided that $n>0$. Hence, for $n \geqq 1$,

$$
\begin{aligned}
2 \pi c_{n}= & i e^{-i n \alpha} \int_{1}^{\infty} f\left(t e^{i \alpha}\right) t^{n-1} d t-i e^{-i n(2 \pi-\alpha)} \int_{1}^{\infty} f\left(t e^{-i \alpha}\right) t^{-n-1} d t \\
& +\int_{\alpha}^{2 \pi-\alpha} e^{-i n \theta} f\left(e^{i \theta}\right) d \theta .
\end{aligned}
$$

Thus for $n \geqq 1$,

$$
\begin{aligned}
(-1)^{n} 2 \pi c_{n}= & i e^{i n(\pi-\alpha)} \int_{1}^{\infty} t^{-n-1} f\left(t e^{i \alpha}\right) d t-i e^{-i(n \pi-\alpha)} \int_{1}^{\infty} t^{-n-1} f\left(t e^{-i \alpha}\right) d t \\
& -\int_{-(\pi-\alpha)}^{\pi-\alpha} f\left(-e^{-i \theta}\right) e^{i n \theta} d \theta .
\end{aligned}
$$

We now write 


$$
\begin{gathered}
F(z)=i e^{i z(x-\alpha)} \int_{1}^{\infty} t^{-z-1} f\left(t e^{i \alpha}\right) d t-i e^{-i z(\pi-\alpha)} \int_{1}^{\infty} t^{z-1} f\left(t e^{-i \alpha}\right) d t \\
-\int_{-(\pi-\alpha)}^{\pi-\alpha} f\left(-e^{-i \theta}\right) e^{i z \theta} d \theta .
\end{gathered}
$$

Then $F(z)$ is analytic for $x>0$,

$$
|F(x+i y)| \leqq A e^{(x-\alpha)|y|}, \quad x \geqq 1,
$$

where $A$ is a constant, and $F\left(\mu_{n}\right)=0$, where $\left\{\mu_{n}\right\}$ is the set of positive integers which are not $\lambda_{n}$ 's. We have

$$
n_{\mu}(r) \geqq((\pi-\alpha) / \pi) r+r \delta(r)+\text { constant. }
$$

By Theorem 2, $F(z) \equiv 0$, and so, in particular, $c_{n}=0$ for $n \geqq 1$. Thus $f(z) \equiv c_{0}$.

As an application of Theorem 9, we can improve a result of Mandelbrojt and Ulrich [6] on quasi-analytic functions. In their Theorem I, the condition $\lim \sup \left(\nu_{m} / m\right)<2$, which can also be written $\lim \sup n_{\nu}(r) / r \geqq 1 / 2$, can be replaced by $n_{\nu}(r)>r / 2+r \delta(r)$, where $\delta(r)$ satisfies the conditions of Theorem 9.

It is natural to seek a decomposition theorem analogous to Theorem 8 . The most obvious one would state that, if $\{n\}=\left\{\lambda_{n}\right\}+\left\{\mu_{n}\right\}$, then, in every angle of opening exceeding $\pi$, one of the series $\sum c_{\lambda_{n}} z^{\lambda_{n}}, \sum c_{\mu_{n}} z^{\mu_{n}}$ has a singularity, is unbounded, or is constant; this is a weaker result than Theorem 8. However, the following two theorems are not contained in Theorem 8 .

THEOREM 10. Let $\left\{c_{n}\right\}$ be any sequence. Let the positive integers be divided into disjoint sets $\left\{{ }_{j} \lambda_{n}\right\}(j=1,2, \cdots, m)$. Let $\alpha_{1}+\cdots+\alpha_{m}>\pi$. Then, if functions $f_{j}(z)$ are defined by the series

$$
f_{j}(z)=\sum_{n=1}^{\infty} c_{j \lambda_{n}} z^{\lambda_{n}}
$$

and if we associate with $f_{j}(z)$ an angle of opening $2 \alpha_{j}$, with vertex at the origin, at least one $f_{j}(z)$ has a singular point, is unbounded, or is constant in the corresponding angle.

Suppose that every $f_{j}(z)$ is analytic and bounded in the corresponding angle, and let $c_{0}=0$. By replacing $f_{j}(z)$ by $f_{j}\left(z e^{i \beta}\right)$ with a suitable $\beta$, we can make the $j$ th angle be $|\theta| \leqq \alpha_{j}$. We then form the functions $F_{j}(z)$ as in the proof of Theorem $9 ; F_{j}(z)$ satisfies

$$
\left|F_{j}(z)\right| \leqq A_{j} e^{\left(x-\alpha_{j}\right)|y|} .
$$

We have $F_{j}\left({ }_{j} \mu_{n}\right)=0$, where the ${ }_{j} \mu_{n}$ are the $n$ 's which are not ${ }_{j} \lambda_{n}$ 's. Let

$$
F(z)=F_{1}(z) F_{2}(z) \cdots F_{m}(z) .
$$


Then $F(z)$ has an $(m-1)$-fold zero at every $n$, and satisfies

$$
|F(z)| \leqq A e^{\left.\mid m \pi-\left(\alpha_{1}+\cdots+\alpha_{m}\right)\right\}|y|} .
$$

If $\pi(m-1)>m \pi-\left(\alpha_{1}+\cdots+\alpha_{m}\right)$, that is, $\alpha_{1}+\cdots+\alpha_{m}>\pi, F(z) \equiv 0$ by Theorem 2 with $k=m \pi-\left(\alpha_{1}+\cdots+\alpha_{m}\right)$; we do not use the full force of Theorem 2. Then one $F_{j}(z)$ at least must vanish identically.

We can obtain a somewhat different result if we start from a sequence which already has gaps.

THEOREM 11. Let $\left\{c_{n}\right\}$ be a sequence in which $c_{n}=0$ except for $n=\lambda_{n}$, where

$$
n_{\lambda}(t) \leqq c t-t \delta(t), \quad c<1,
$$

with the same conditions on $\delta(t)$ as in Theorem 9. If $\left\{\lambda_{n}\right\}$ is divided into $m$ disjoint sequences $\left\{{ }_{k} \lambda_{n}\right\}(k=1, \cdots, m ; m>1)$ and $f_{k}(z)$ are the corresponding functions, then in every angle of opening $2 \pi c(1-1 / m)$, with vertex at $z=0$, at least one of $f_{k}(z)$ has a singular point, is unbounded, or is a constant.

We suppose that all the $f_{k}(z)$ are analytic and bounded in the angle $|\theta| \leqq \alpha$ and form functions $F_{k}(z)$ as in the proof of Theorem 9. Let $F(z)=F_{1}(z) \cdots F_{m}(z)$. Let $\left\{\mu_{n}\right\}$ be the sequence of integers which are not $\lambda_{n}$ 's. Then $F\left(\lambda_{n}\right)=0$ for every $n$, and in addition $F(z)$ has an $m$-fold zero at each $\mu_{n}$. Hence the number of zeros of $F(z)$ in $(0, t)$ is

$$
\begin{aligned}
t+(m-1)\{(1-c) t & +t \delta(t)\}+O(1) \\
& =\{m(1-c)+c\} t+(m-1) t \delta(t)+O(1) .
\end{aligned}
$$

On the other hand, $\log \left|F_{k}(z)\right|=O\left(e^{(x-\alpha)|y|}\right)$, and so

$$
\log |F(z)|=O\left(e^{m(x-\alpha)|y|}\right) \text {. }
$$

By Theorem 2, $F(z) \equiv 0$, and so some $F_{k}(z) \equiv 0$, provided that

$$
\pi\{m(1-c)+c\} \geqq m(\pi-\alpha),
$$

or $\alpha \geqq \pi c(1-1 / m)$.

\section{REFERENCES}

1. R. P. Agnew, On sequences with vanishing even or odd differences, Amer. J. Math. vol. 66 (1944) pp. 339-340.

2. R. P. Boas, Jr., On a generalization of the Stieltjes moment problem, Trans. Amer. Math. Soc. vol. 46 (1939) pp. 142-150.

2a. R. P. Boas, Jr., and H. Pollard, Properties equivalent to the completeness of $\left\{e^{-t} t^{\lambda_{n}}\right\}$, Bull. Amer. Math. Soc. vol. 52 (1946) pp. 348-351.

3. W. H. J. Fuchs, $A$ theorem on finite differences with an application to the theory of Hausdorff summability, Proc. Cambridge Philos. Soc. vol. 40 (1944) pp. 189-197.

3a. - On the closure of $\left\{e^{-t t_{\nu}}\right\}$, Proc. Cambridge Philos. Soc. vol. 42 (1946) pp. 91-105. 
4. S. Mandelbrojt, Series lacunaires, Actualites Scientifiques et Industrielles, no. 305, Hermann, Paris, 1936.

5. - Dirichlet series, Rice Institute Pamphlet, vol. 31 (1944) pp. 159-272.

6. S. Mandelbrojt and F. E. Ulrich, On a generalization of the problem of quasi-analyticity, Trans. Amer. Math. Soc. vol. 52 (1942) pp. 265-282.

7. R. E. A. C. Paley and N. Wiener, Fourier transforms in the complex domain, Amer. Math. Soc. Colloquium Publications, vol. 19, New York, 1934.

8. H. Pollard, Sequences with vanishing even differences, Duke Math. J. vol. 12 (1945) pp. 303-304.

9. J. A. Shohat and J. D. Tamarkin, The problem of moments, Mathematical Surveys, vol. 1, American Mathematical Society, New York, 1943.

10. E. C. Titchmarsh, The theory of functions, Oxford University Press, 1932.

Brown UNIVERSITY,

PRovidence, R.I. 